\title{
David-V Procedure in a Patient with Aortic Dilation and Competent Quadricuspid Aortic Valve: Are Genetics to Blame?
}

Katherine R. Hebeler, BA, John J. Squiers, BSE, Heike Baumgarten, MD, J. Michael DiMaio, MD, William T. Brinkman, MD*

Department of Cardiothoracic Surgery, The Heart Hospital Baylor Plano, Plano, Texas, USA

\begin{abstract}
Quadricuspid aortic valves (QAVs) are extremely rare. In this case study, we report a David-V valve-sparing aortic root replacement with reimplantation of a native QAV in a patient with aortic dilation, normal valve function, and a family history of aortic dissection. Microscopic pathological examination of the excised section of the aorta revealed scattered small foci of cystic medical degeneration throughout. A genetic predisposition for aortic dilation may be present in patients with QAV, even in the setting of a competent valve. Regular screening for aortic dilation in patients with known QAV should therefore be considered.

Copyright (c) 2016 Science International Corp.
\end{abstract}

\section{Key Words}

Quadricuspid aortic valve • Aortic dilation • Genetics

\section{Introduction}

First described by Babington in 1847 [1], quadricuspid aortic valve (QAV) is a rare congenital anomaly with an estimated prevalence of $0.003-0.04 \%$ in the general population $[2,3]$. QAV associated with aortic regurgitation or stenosis commonly requires replacement or repair in the fifth or sixth decade of life [4]. Ascending aortic aneurysm has historically been reported only rarely in cases of QAV, but two recent

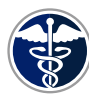

Fax +1 2037853552

E-Mail: aorta@scienceinternational.org

http://aorta.scienceinternational.org

\author{
(c) 2016 AORTA \\ Published by Science International Corp. \\ ISSN 2325-4637 \\ Accessible online at: \\ http://aorta.scienceinternational.org
}

series published from the Cleveland Clinic and Mayo Clinic identified aortic dilation in $30-40 \%$ of patients with QAV $[5,6]$. However, whether aortic dilation associated with QAV is genetically or hemodynamically mediated remains controversial [7]. We report a David-V valve-sparing aortic root replacement with reimplantation of a native QAV in a patient with aortic dilation and normal valve function. The patient's presentation, operative findings, and pathological analysis suggest that a genetic predisposition for aortic dilation may be present in some patients with QAV.

\section{Case Presentation}

A 47-year-old white male with a family history of aortic dissection presented with new onset chest pain and dyspnea exacerbated by exertion. The patient reported exercising regularly with no dyspnea at rest. The patient's brother had previously died from an aortic dissection.

Computed tomography of the chest revealed an aneurysm in the ascending aorta with a dilated aortic root diameter of $4.5 \mathrm{~cm}$ maximally. Dilation of the proximal and mid-ascending aorta was observed and measured $4.0 \mathrm{~cm}$ in diameter maximally for a length of $7.0 \mathrm{~cm}$. Transthoracic echocardiography (TTE) confirmed the aneurysm. No aortic regurgitation or aortic stenosis was visualized by TTE, and the left ventricular

\footnotetext{
* Corresponding Author:

William T. Brinkman, MD

Department of Cardiothoracic Surgery

The Heart Hospital Baylor Plano

1100 Allied Drive, Plano, TX, USA

Tel.: +1 469814 4720; Fax: +1 469814 4854; E-Mail: William.brinkman@bswhealth.org
} 
ejection fraction was $55-65 \%$. Given the patient's strong family history, prophylactic surgical aortic root and ascending aortic replacement was planned.

A David- $V$ valve-sparing aortic root replacement was performed via median sternotomy on cardiopulmonary bypass. Preoperative echocardiography demonstrated a tricuspid aortic valve, but the patient was found to have QAV in the operating room. A small $1-\mathrm{cm}$ cusp at the left non-commissure area with two separate commissural posts was visualized (Type B QAV; Figure 1) [2]. Upon further inspection, the valve was functioning well. Therefore, the native QAV was reimplanted with the two commissural posts suspended together at the left non-commissure area (Figure 2).

A $30-\mathrm{mm}$ aortic graft with a prefabricated sinus segment was trimmed to size, and subannular sutures were placed through the bottom of the Valsalva portion of the graft. Sizing of the graft was estimated from the commissural height at the left non-commissure. The commissure posts were resuspended at the neosinotubular junction. The valve remained competent and was then suspended and sewn into the Valsalva portion of the graft. The left and right coronary buttons were sewn into the graft, which was then

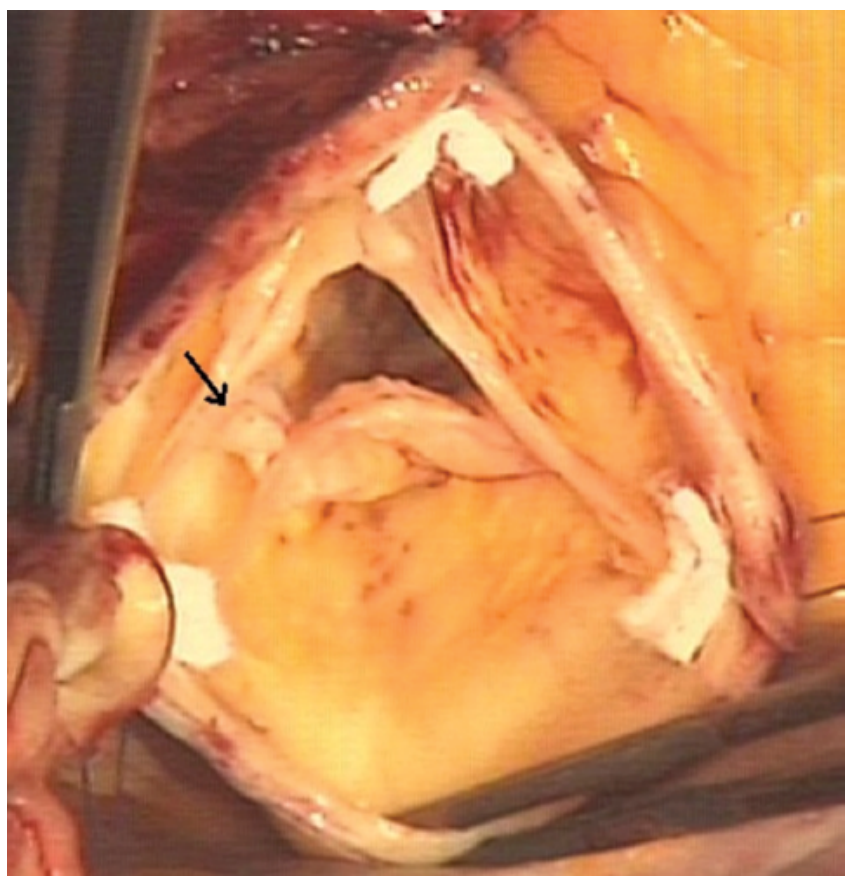

Figure 1. Intraoperative view of the quadricuspid aortic valve. The fourth leaflet is indicated by the arrow. sewn into the distal ascending aorta. The patient was weaned from cardiopulmonary bypass without difficulty. Trivial aortic insufficiency was detected at the time of closure (Figure 3). The patient tolerated the procedure well and was discharged from the hospital on the third postoperative day.

Microscopic pathological examination of the excised section of aorta revealed focal laminar necrosis with scattered foci of cystic medial degeneration throughout. No significant inflammation was present.

\section{Discussion}

Two recently published case series that described patients with surgically treated QAV and patients with QAV identified from an echocardiographic database reported a high prevalence of aortic root and ascending aortic dilation in patients with QAV, suggesting the two abnormalities may share a pathophysiologic link $[5,6]$. Whether the aortic dilation
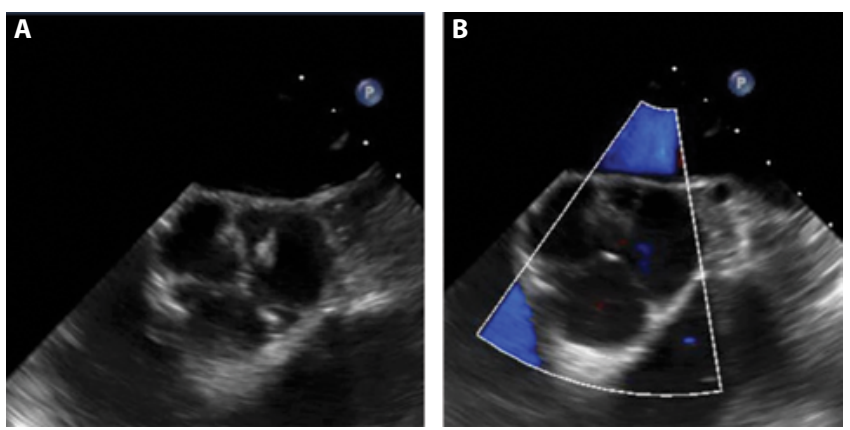

Figure 2. Panel A. Intraoperative transesophageal echocardiography depicts a quadricuspid aortic valve. Panel B. Color Doppler image demonstrates valve competence.
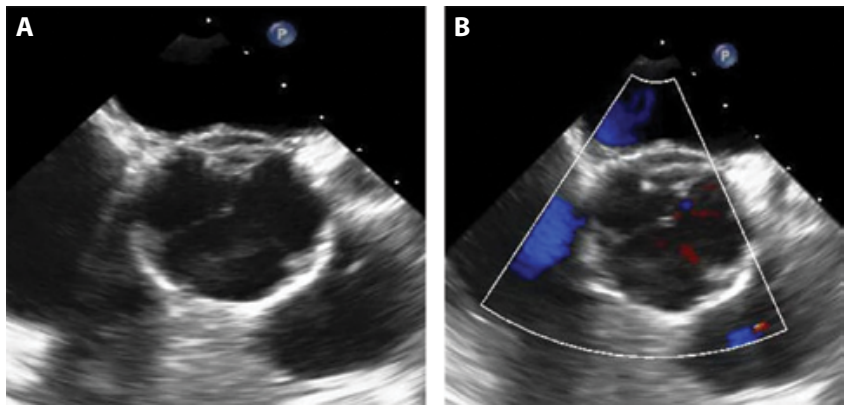

Figure 3. Panel A. Transesophageal echocardiography shows continued competence of the quadricuspid aortic valve after reimplantation. Panel B. Color Doppler confirms valve competence. 
observed in patients with QAV is the result of a genetically mediated aortopathy, altered hemodynamic factors associated with the abnormal valve, or both is unknown [7]. Given our patient's family history of dissection, the lack of aortic valve dysfunction, and the cystic medial necrosis identified by pathology analysis, we postulate that an unidentified genetic etiology that links QAV with aortic dilation may be present in some patients, including the one described herein, although no other signs of connective tissue disorder were noted in the medical history or physical exam and definitive genetic testing was not performed [8]. Although the patient's brother's aortic valve morphology was unknown and focal laminar necrosis, which is associated with acquired degeneration of the aortic wall, was identified by pathology in addition to cystic medial necrosis, our patient's case nevertheless offers some limited insight into the etiology of aortic dilation associated with QAV. Because a genetic predisposition for aortic dilation may be present in patients with QAV, regular screening for aortic dilation in this population should be considered.

\section{Conflict of Interest}

The authors have no conflict of interest relevant to this publication.

\section{Comment on this Article or Ask a Question}

\section{References}

1. Babington BG. Case of cyanosis dependent patent ductus arteriosus. London Medical Gazette. 1847;4:822-823.

2. Hurwitz LE, Roberts WC. Quadricuspid semilunar valve. Am J Cardiol. 1973;31:623626. DOI: 10.1016/0002-9149(73)90332-9

3. Uspenskiy VE, Osadchii AM, Gordeev M. Quadricuspid aortic valve combined with moderate ascending aortic dilatation: a report of four cases. AORTA (Stamford). 2015;3:187-190. DOI: 10.12945/j.aorta.2015.15.004

4. Jagannath $A D$, Johri $A M$, Liberthson R, Larobina M, Passeri J, Tighe D, et al. Quadricuspid aortic valve: a report of 12 cases and a review of the literature. Echocardiography. 2011;28:1035-1040. DOI: 10.1111/j.15408175.2011.01477.x
5. Idrees IJ, Roselli EE, Arafat A, Johnston DR, Svensson LG, Sabik JF 3rd, et al. Outcomes after repair or replacement of dysfunctional quadricuspid aortic valve. J Thorac Cardiovasc Surg. 2015;150:79-82. DOI: 10.1016/j.jtcvs.2015.03.019

6. Tsang MYC, Abudiab MM, Ammash NM, Nagvi TZ, Edwards WD, Nkomo VT, et al. Quadricuspid aortic valve: Characteristics, associated structural cardiovascular abnormalities, and clinical outcomes. Circulation. 2016;133:312-319. DOI: 10.1161/ CIRCULATIONAHA.115.017743

7. Tsukioka $\mathrm{K}$, Nobara $\mathrm{H}$, Takano $\mathrm{T}$, Wada $\mathrm{Y}$, Amano J. Quadricuspid aortic valve with ascending aortic aneurysm: a case report and histopathological investigation. Ann Thorac Cardiovasc Surg. 2011;17:418-421. DOI: 10.5761 /atcs.cr.10.01567
8. Marsalese DL, Moodie DS, Lytle BW, Cosgrove DM, Ratliff NB, Goormastic M, et al. Cystic medial necrosis of the aorta in patients without Marfan's syndrome: Surgical outcome and long-term follow-up. J Am Coll Cardiol. 1990;16:68-73. DOI: 10.1016/0735-1097(90)90458-2

Cite this article as: Hebeler KR, Squiers JJ, Baumgarten H, DiMaio JM, Brinkman WT. David-V Procedure in a Patient with Aortic Dilation and Competent Quadricuspid Aortic Valve: Are Genetics to Blame? AORTA (Stamford). 2016;4(5):178-180. DOI: http://dx.doi. org/10.12945/j.aorta.2016.16.033 